\title{
From population to individuals: a new indicator for evaluating the appropriateness of clinical application of antibiotics
}

\author{
Bin Zheng ${ }^{1+}, \mathrm{Na} \mathrm{Li}^{1+}$, Zhijian $\mathrm{Hu}^{2}$ and Maobai Liu ${ }^{1 *}$
}

\begin{abstract}
Background: This study aims to establish a new indicator based on the anatomical therapeutic chemical/defined daily dose (ATC/DDD) system.

Methods: Utilization data of antibiotics of inpatients in a university hospital were used to calculate the indicators of use rate (UR), use density (UD), and ratio of use density to use rate (UD/UR). According to the professional characteristics, the recommended values of UD/UR in different departments were established respectively. Crosswise comparison and appropriateness evaluation between different treatment groups with the same profession were performed. For individual inpatients with abnormally increased drug utilization index (DUI) and ratios of antimicrobial course to length of stay $(C / S)$, detailed analysis was performed to examine whether any irrational drug utilization occurred.

Results: The indicator UD/UR combines both dose and duration of treatment, which were the two main factors affecting the appropriateness of clinical application of antibiotics. Thus, it can more sensitively reveal the drug utilization of inpatients receiving antibiotics. UD/UR is also more suitable for evaluating the clinical appropriateness of antibiotic application than the macroscopic indicator, total UD, and could be applied at the macroscopic and microscopic levels.
\end{abstract}

Conclusions: The ratio UD/UR has great practical value and can serve as a reference for evaluating the appropriateness of clinical application of antibiotics.

Keywords: Defined daily dose (DDD), Antibiotics, Ratio of use density to use rate (UD/UR), Appropriateness evaluation, Indicator

\section{Background}

Irrational antibiotic utilization, the most important cause of the antibiotic resistance of bacteria, is a problem requiring effective supervision in the clinical application of antibiotics. The Anatomical Therapeutic Chemical (ATC) Classification System and the measurement unit of defined daily dose (DDD) [1] were recommended and officially regarded by the World Health Organization

\footnotetext{
* Correspondence: liumaobai@163.com

${ }^{\dagger}$ Bin Zheng and $\mathrm{Na}$ Li contributed equally to this work.

${ }^{1}$ Department of Pharmacy, Fujian Medical University Union Hospital, 29 Xinquan Road, Gulou District, Fuzhou City 350001, Fujian Province, China Full list of author information is available at the end of the article
}

(WHO) as the international standard in drug utilization research (DUR) in 1996. The number of DDDs [2] and drug use density (DDDs per patient day [3], DDDs per 100 patient days [3-6], DDDs per 1000 patient days [7-10], DDDs per admission [3, 11], DDDs per 1000 admissions [3, 12, 13], or DDDs per operation [14]) established based on the ATC/DDD system have been widely used as important indicators of antibiotic utilization research. However, although they can be obtained easily and applied to continuously and systematically monitor the administration of antibiotics in a population, these macro-indicators are not meaningful in evaluating the appropriateness of drug utilization. In

(c) The Author(s). 2018 Open Access This article is distributed under the terms of the Creative Commons Attribution 4.0 International License (http://creativecommons.org/licenses/by/4.0/), which permits unrestricted use, distribution, and reproduction in any medium, provided you give appropriate credit to the original author(s) and the source, provide a link to the Creative Commons license, and indicate if changes were made. The Creative Commons Public Domain Dedication waiver (http://creativecommons.org/publicdomain/zero/1.0/) applies to the data made available in this article, unless otherwise stated. 
addition, establishing rigid standards is difficult. Consideration of the specific condition of each patient is required when micro-indicators such as indication, selection of variety, prophylactic administration time before operation, dose, frequency and course of administration are used for the appropriateness evaluation of clinical application of antibiotics. Unfortunately, in spite the high precision, strong expertise and long processing times are required to evaluate with these micro-indicators; thus, the feasibility of their application to bulk operation is poor. Similar to macro-indicators, establishing uniform standards for these micro-indicators is difficult [15]. Therefore, a more suitable indicator for the appropriateness evaluation of clinical application of antibiotics that could be adapted to both populations and individuals, must be developed. This article proposes a new indicator, i.e., ratio of use density to use rate (UD/UR), for the appropriateness evaluation of antibiotics and investigates its application at the macroscopic and microscopic levels.

\section{Methods}

\section{Materials}

The research materials included utilization data of antibiotics in a 2500-bed university hospital. The name, specification, dose, frequency, and course, among other information, of antibiotics used by inpatients in each department in a certain month were obtained from the Hospital Information System (HIS). All study protocols were performed in accordance with and approved by the Committee of Research Ethics of Fujian Medical University Union Hospital (2018KY013, Fuzhou, China).

\section{Methods}

\section{Derivation of equation}

According to Management of Medical Quality and Control Indicators of Tertiary General Hospitals (version 2011) published by the National Health and Family Planning Commission (NHFPC) of the People's Republic of China [16], the UR and UD of antibiotics used by inpatients in each department can be calculated using eqs. $1,2,3$. Equation (4 can be derived from eqs. 1, 2, 3 to calculate the UD/UR in each department. Equation (9 can be derived from eq. 5 of the drug utilization index (DUI) and self-defined eqs. 6, 7, 8 .

UR (proportion of inpatients receiving antibiotics) (\%)

$=$ number of inpatients receiving antibiotics

/ total number of inpatients $\times 100 \%$

$$
\begin{aligned}
\mathrm{UD} & =\sum[\text { consumption of antibiotics }(\mathrm{g}) / \mathrm{DDD}(\mathrm{g} / \text { day })] \\
& / \text { number of patient days } \times 100 \\
& =(\text { number of DDDs } \times 100) / \text { number of patient days }
\end{aligned}
$$

$$
\begin{aligned}
\text { Number of patient days } & =\text { total number of inpatients } \\
& \times \text { mean length of stay of inpatients }
\end{aligned}
$$

$$
\begin{aligned}
\mathrm{UD} / \mathrm{UR}= & {[\text { number of DDDs } \times 100] } \\
& /[\text { number of inpatients receiving antibiotics } \\
& \times \text { mean length of stay of inpatients }]
\end{aligned}
$$

DUI $=$ number of DDDs/total medication days

Total medication days

$$
\begin{aligned}
& =\text { number of inpatients receiving antibiotics } \\
& \times \text { mean treatment days of inpatients receiving antibiotics }
\end{aligned}
$$

Ratio of antimicrobial course to length of stay $(\mathrm{C} / \mathrm{S})$

$=$ mean treatment days of inpatients receiving antibiotics

/ mean length of stay of inpatients receiving antibiotics

Elongation factor of length of stay (EF)

$=$ mean length of stay of inpatients receiving antibiotics

/ mean length of stay of inpatients

$$
\mathrm{UD} / \mathrm{UR}=\mathrm{DUI} \times(\mathrm{C} / \mathrm{S}) \times \mathrm{EF} \times 100
$$

The UD, UR, and UD/UR of antibiotics of inpatients in each department were calculated. Based on the professional characteristics, the recommended values of UD/UR of some departments were established respectively.

The UD/UR of antibiotics of inpatients in different treatment groups with the same profession were calculated, and crosswise comparison and appropriateness evaluation were performed.

The UD/UR of the antibiotics of individual inpatients were calculated. Patients with abnormally increased DUI or $\mathrm{C} / \mathrm{S}$ were identified, and their electronic medical records were studied to further examine whether any irrational drug utilization had occurred. 


\section{Results}

The National Program of Special Renovation Activity on the Clinical Application of Antibiotics published by the NHFPC in 2012 [17] states that "the UR should be no more than $60 \%$ and the UD should be controlled under 40 DDDs per 100 patient days in general hospitals". In this research, the UD exceeded 40 DDDs in 17 departments and the UR exceeded $60 \%$ in 11 departments (Table 1). However, the UD were less than 40 DDDs and the UR exceeded $60 \%$ in both the geriatrics and gynecology departments. By contrast, the UD exceeded 40 DDDs and the UR were less than $60 \%$ in the departments of hematology, general surgery, nephrology, rheumatology, neurology, and burns. The UR and UD of antibiotics in these departments did not synchronously reach the recommended standards, thereby indicating that the results of the appropriateness evaluation of clinical application of antibiotics would be limited if such evaluation was performed using only these two indicators.

According to eq. 9, given that it was positively correlated with the product of DUI, C/S, and EF, UD/UR could be decomposed into three terms for individual analysis. DUI is usually applied as a standard to judge the appropriateness of clinical medication $[18,19]$. For a single variety of antibiotics, DUI $>1.0$ means the prescribed daily dose (PDD) of the drug is more than the DDD, indicating the possibility of overdose. DUI $\leq 1.0$ means the PDD of the drug is less than or equal to its DDD, indicating that the dose is rational [20]. Hence, different recommended values of DUI can be established according to the professional characteristics of each department. For departments such as hematology, respiratory care, or intensive care unit, inpatients are usually prescribed with therapeutic purpose and receive combined utilization of two to three types of different antibiotics; thus, the rational value of DUI can be assigned as 2 or 3. For departments such as thyroid gland surgery or breast surgery, inpatients are usually prescribed with a single type of prophylactic antibiotics; thus, the rational value of DUI can be assigned as 1 . Different recommended values of $\mathrm{C} / \mathrm{S}$ can also be established according to the professional characteristics of each department. For therapeutic departments, such as hematology, respiratory care, or intensive care unit, antimicrobial courses usually last from admission to hospital discharge; thus, the rational ratio can be set as 1 . For preventative departments, such as thyroid gland surgery or breast surgery, the duration of prophylactic antibiotics is usually less than or equal to 1 day ( $24 \mathrm{~h}$ ); thus, the rational ratio can be set as "one day to the mean length of stay of inpatients receiving antibiotics". The EF reflects the extension degree of the mean length of stay of inpatients receiving antibiotics compared with all inpatients and its rational value is usually set as 1 . Considering DUI, C/S, and EF, the recommended values of UD/UR of antibiotics of inpatients in some departments can be obtained (Table 2).

In Table 1, the UD/UR of departments of thyroid surgery and breast surgery were $>100$, indicating a high possibility of irrational medication and requirement of enhanced monitoring. However, the UR and UD of antibiotics in these two departments reached the recommended standards with extremely low values. This finding reveals that UD/UR has high sensitivity in the appropriateness evaluation of clinical antibiotics application. UD/UR was $>300$ in the department of hematology and were $>200$ in the departments of general surgery, oncology, infectious diseases, and respiratory care, which indicates a possibility of irrational medication and requirement of enhanced monitoring.

In Table 3, crosswise comparison revealed that UD/UR was obviously higher in group $\mathrm{G}$ of the oncology department, group I of the hematology department, group A of the respiratory care department, group B of the rheumatology department, group A of the cardiology department, group $\mathrm{C}$ of the neurology department, group A of the endocrinology department, group A of the ophthalmology department, group A of the gastrointestinal surgery department, group A of the neurosurgery department, group $\mathrm{C}$ of the burn department, group $\mathrm{C}$ of the stomatology department, group $C$ of the hepatobiliary surgery department, group $\mathrm{B}$ of the cardiac surgery department, group B of the interventional treatment department, and group $C$ of the breast surgery department than in other groups with the same profession. Thus, enhanced monitoring is required in these groups.

UD/UR was $>300$ in groups B, F, G, H, and I of the hematology department; It was $>200$ in groups D, F, and $\mathrm{G}$ of the oncology department; group A of the respiratory care department; and group B of the rheumatology department; It was $>100$ in groups A, D, and F of the cardiology department; group A of the ophthalmology department; group A of the gastrointestinal surgery department; groups $\mathrm{A}, \mathrm{B}, \mathrm{D}$, and $\mathrm{E}$ of the neurosurgery department; group $\mathrm{C}$ of the stomatology department; group $\mathrm{B}$ of the interventional treatment department; and groups $\mathrm{C}$ and $\mathrm{D}$ of the breast surgery department. These findings indicate a possibility of severe infection or irrational medication. Hence, enhanced monitoring is required in these groups.

For individual inpatients, $\mathrm{UR}=100 \%$ and $\mathrm{EF}=1$. eq. (9) can be written as UD $=$ DUI $\times(C / S) \times 100(10)$. Based on this equation, the brief chart in Table 4 was designed in the HIS in coordination with the information department of the hospital. Autonomous statistics can be achieved at any time to identify patients with abnormally increased DUI and C/S. In addition, the brief chart can be linked to 
Table 1 Antibacterial utilization of inpatients in each department

\begin{tabular}{|c|c|c|c|c|c|c|}
\hline Ranking & Department & UR & Department & UD & Department & UD/UR \\
\hline 1 & Infectious diseases & $100.00 \%$ & Infectious diseases & 220.00 & Hematology & 348.04 \\
\hline 2 & Intensive care unit & $88.89 \%$ & Respiratory care & 170.33 & Pediatric hematology & 291.12 \\
\hline 3 & Respiratory care & $81.19 \%$ & Intensive care unit & 136.91 & General surgery & 249.30 \\
\hline 4 & Emergency surgery & $79.35 \%$ & Emergency surgery & 136.55 & Oncology & 224.47 \\
\hline 5 & Ear-nose-throat & $77.50 \%$ & Hematology ${ }^{b}$ & 113.67 & Infectious diseases & 220.00 \\
\hline 6 & Emergency medicine & $72.73 \%$ & Emergency medicine & 101.52 & Respiratory care & 209.79 \\
\hline 7 & Neonatology & $71.05 \%$ & Cardiac surgery & 72.81 & Neurology & 197.49 \\
\hline 8 & Cardiac surgery & $67.46 \%$ & Ear-nose-throat & 67.99 & Emergency surgery & 172.09 \\
\hline 9 & Gynecology $^{a}$ & $66.78 \%$ & Pediatric hematology & 65.24 & Neurosurgery & 162.62 \\
\hline 10 & geriatrics $^{a}$ & $65.79 \%$ & General surgery $^{\mathrm{b}}$ & 64.32 & Intensive care unit & 154.02 \\
\hline 11 & Stomatology & $64.96 \%$ & Stomatology & 53.39 & Interventional therapy & 146.22 \\
\hline 12 & Urology & $59.71 \%$ & Nephrology ${ }^{b}$ & 51.59 & Pediatrics & 143.01 \\
\hline 13 & Pediatric surgery & $57.14 \%$ & Hepatobiliary surgery & 51.57 & Emergency medicine & 139.58 \\
\hline 14 & Hepatobiliary surgery & $54.47 \%$ & Pediatrics & 49.48 & Cardiology & 136.92 \\
\hline 15 & Nephrology ${ }^{\mathrm{b}}$ & $43.24 \%$ & Rheumatology ${ }^{b}$ & 44.7 & Thyroid gland surgery & 129.55 \\
\hline 16 & Burns $^{b}$ & $41.67 \%$ & Neurology ${ }^{b}$ & 40.88 & Rheumatology & 124.17 \\
\hline 17 & Orthopedics & $40.91 \%$ & Burns $^{b}$ & 40.47 & Endocrinology & 123.20 \\
\hline 18 & Gastroenterology & $38.92 \%$ & Urology & 37.62 & Nephrology & 119.31 \\
\hline 19 & Rheumatology $^{\mathrm{b}}$ & $36.00 \%$ & Neurosurgery & 35.89 & Colorectal surgery & 114.77 \\
\hline 20 & Thoracic surgery & $35.58 \%$ & Thoracic surgery & 34.63 & Cardiac surgery & 107.93 \\
\hline 21 & Pediatrics & $34.60 \%$ & Pediatric surgery & 33.52 & Breast surgery & 103.33 \\
\hline 22 & Hematology ${ }^{b}$ & $32.66 \%$ & geriatrics $^{a}$ & 31.39 & Thoracic surgery & 97.33 \\
\hline 23 & Obstetrics & $32.34 \%$ & Neonatology & 31.06 & Burns & 97.12 \\
\hline 24 & Gastrointestinal surgery & $30.60 \%$ & Colorectal surgery & 30.38 & Hepatobiliary surgery & 94.68 \\
\hline 25 & Colorectal surgery & $26.47 \%$ & Gastroenterology & 29.78 & Ear-nose-throat & 87.73 \\
\hline 26 & General surgery ${ }^{b}$ & $25.80 \%$ & Orthopedics & 27.71 & Traditional Chinese medicine & 87.35 \\
\hline 27 & Radio-oncology & $23.57 \%$ & Cardiology & 26.11 & Stomatology & 82.19 \\
\hline 28 & Pediatric hematology & $22.41 \%$ & Gynecology $^{a}$ & 25.39 & Gastrointestinal surgery & 81.90 \\
\hline 29 & Neurosurgery & $22.07 \%$ & Gastrointestinal surgery & 25.06 & Gastroenterology & 76.52 \\
\hline 30 & Traditional Chinese medicine & $21.74 \%$ & Endocrinology & 21.19 & Orthopedics & 67.73 \\
\hline 31 & Neurology $y^{b}$ & $20.70 \%$ & Oncology & 19.08 & Radio-oncology & 65.21 \\
\hline 32 & Cardiology & $19.07 \%$ & Traditional Chinese medicine & 18.99 & Urology & 63.00 \\
\hline 33 & Plastic surgery & $18.86 \%$ & Radio-oncology & 15.37 & Pediatric surgery & 58.66 \\
\hline 34 & Endocrinology & $17.20 \%$ & Obstetrics & 13.81 & Rehabilitation & 57.61 \\
\hline 35 & Day care ward & $12.15 \%$ & Interventional treatment & 8.13 & Day care ward & 57.37 \\
\hline 36 & Oncology & $8.50 \%$ & Plastic surgery & 7.88 & geriatrics & 47.71 \\
\hline 37 & Rehabilitation & $7.69 \%$ & Day care ward & 6.97 & Neonatology & 43.72 \\
\hline 38 & Intervention treatment & $5.56 \%$ & Thyroid gland surgery & 5.48 & Obstetrics & 42.70 \\
\hline 39 & Thyroid gland surgery & $4.23 \%$ & Rehabilitation & 4.43 & Plastic surgery & 41.78 \\
\hline 40 & Ophthalmology & $1.31 \%$ & Breast surgery & 0.93 & Ophthalmology & 38.93 \\
\hline 41 & Breast surgery & $0.90 \%$ & Ophthalmology & 0.51 & Gynecology & 38.02 \\
\hline
\end{tabular}


Table 2 Recommended values of UD/UR of antibiotics of inpatients in some departments

\begin{tabular}{|c|c|c|c|c|c|}
\hline \multirow[t]{2}{*}{ Department } & & \multicolumn{4}{|c|}{ Recommended values } \\
\hline & & $\overline{\mathrm{DUI}}$ & $C / S$ & $E F$ & UD/UR \\
\hline \multirow[t]{3}{*}{ Therapeutic department } & Hematology & 3 & 1 & 1 & 300 \\
\hline & Respiratory care & 2 & 1 & 1 & 200 \\
\hline & Intensive care unit & 2 & 1 & 1 & 200 \\
\hline \multirow[t]{2}{*}{ Preventative department } & Thyroid gland surgery & 1 & $1 /\left(9.15^{\mathrm{a}}\right)$ & 1 & 10.93 \\
\hline & Breast surgery & 1 & $1 /\left(5.77^{\mathrm{b}}\right)$ & 1 & 17.33 \\
\hline
\end{tabular}

${ }^{a}$ Mean length of stay of inpatients receiving antibiotics in the department of thyroid gland surgery

${ }^{\mathrm{b}}$ Mean length of stay of inpatients receiving antibiotics in the department of breast surgery

a patient's electronic medical records, thereby enabling detailed analysis on the rationality of medication.

For example, DUI of patient $78 \times \times 89$ was relatively high. Review of the patient's electronic medical records showed subarachnoid hemorrhage and aneurysm of the posterior communicating artery. On January 24, intracranial aneurysm clipping was performed. Ceftriaxone was administered at a dosage of $3 \mathrm{~g}$ per day (qd) through intravenous infusion (ivgtt) for surgical prophylaxis from January 24 to January 26. The Indications and Usage section [21] specifies that for surgical prophylaxis, a single $1 \mathrm{~g}$-dose of ceftriaxone administered ivgtt $0.5-2 \mathrm{~h}$ before operation is recommended. Thus, the dose of $3 \mathrm{~g}$ qd was too high in this case.

C/S of patient $77 \times \times 74$ was also relatively high. Review of the patient's electronic medical records showed that the patient underwent open reduction and internal fixation for a complex mandibular fracture on January 3. A dose of $2 \mathrm{~g}$ of cefathiamidine was offered three times per day (tid) through ivgtt from January 3 to January 5 .
The duration of prophylactic antibiotics applied to this operation should be no more than 24 h [22]. However, the antimicrobial course of this case was $72 \mathrm{~h}$, which was excessively long.

$\mathrm{C} / \mathrm{S}$ of patient $78 \times \times 09$ was $>0$. Review of the patient's electronic medical records showed treatment by hernioplasty for an inguinal hernia on January 15 and administration of $2 \mathrm{~g}$ of cefathiamidine tid through ivgtt on that day. However, prophylactic administration of antibiotics is not recommended for such an operation $[23,24]$. Thus, application of preventative antibiotics was irrational in this case.

\section{Discussion}

DDD is the most commonly used measurement unit recommended by the WHO for DUR [25-28], and several studies have focused on its differentiation in the clinical setting. Kritsotakis et al. [29] stratified the UR and UD of antibiotics according to clinical service, which allowed areas of concern to be specified and targeted antibiotic

Table 3 UD/UR of antibiotics of inpatients in each treatment group of some departments

\begin{tabular}{|c|c|c|c|c|c|c|c|c|c|c|}
\hline Department & Group A & Group B & Group C & Group D & Group E & Group F & Group G & Group H & Group I & $\overline{\text { Group J }}$ \\
\hline Oncology & 199.39 & 99.96 & 58.18 & 345.43 & 10.81 & 372.64 & 437.28 & 0.00 & & \\
\hline Hematology & 252.42 & 487.37 & 274.90 & 183.90 & 228.55 & 304.81 & 333.36 & 307.78 & 519.13 & 190.67 \\
\hline Respiratory care & 269.39 & 174.31 & 192.60 & 172.77 & & & & & & \\
\hline Rheumatology & 114.60 & 331.25 & 197.56 & & & & & & & \\
\hline Cardiology & 143.28 & 37.17 & 52.75 & 136.19 & 48.45 & 124.87 & 85.10 & & & \\
\hline Neurology & 129.81 & 85.27 & 157.10 & & & & & & & \\
\hline Endocrinology & 137.44 & 98.13 & 81.61 & 121.60 & & & & & & \\
\hline Ophthalmology & 113.39 & 14.51 & & & & & & & & \\
\hline Gastrointestinal surgery & 139.02 & 40.55 & 48.93 & & & & & & & \\
\hline Neurosurgery & 259.62 & 138.40 & 68.47 & 127.31 & 215.94 & 15.79 & & & & \\
\hline Burns & 38.23 & 104.79 & 123.05 & & & & & & & \\
\hline Stomatology & 35.29 & 51.00 & 111.80 & & & & & & & \\
\hline Hepatobiliary surgery & 89.35 & 127.85 & 156.00 & 52.62 & & & & & & \\
\hline Cardiac surgery & 37.22 & 193.25 & 129.98 & 54.78 & 78.90 & 131.08 & 49.34 & 69.98 & 54.62 & 65.98 \\
\hline Interventional treatment & 0.00 & 139.23 & 71.59 & & & & & & & \\
\hline Breast surgery & 0.00 & 38.41 & 459.63 & 227.82 & & & & & & \\
\hline
\end{tabular}


Table 4 Brief chart of antibacterial utilization of individual inpatients

\begin{tabular}{|c|c|c|c|c|c|c|c|}
\hline Record number & Treatment group & Treatment days & Length of stay & DDDs & DUI & $\mathrm{C} / \mathrm{S}$ & UD \\
\hline $78 \times \times 89$ & Group $\times$ of neurosurgery department & 3.00 & 3.00 & 4.50 & 1.50 & 1.00 & 150.00 \\
\hline $77 \times \times 74$ & Group $\times$ of stomatology department & 3.00 & 6.00 & 6.00 & 2.00 & 0.50 & 100.00 \\
\hline $78 \times \times 09$ & Group $\times$ of urology department & 1.00 & 12.00 & 2.00 & 2.00 & 0.08 & 16.67 \\
\hline
\end{tabular}

policy changes to be initiated. A number of disease-specific quality indicators have also been established. For instance, the range of UR of antibiotics for acute cystitis should be $80-100 \%$ while that of quinolones should be $0-5 \%$, as recommended by the European Surveillance of Antimicrobial Consumption [30]. Some researchers have investigated other indicators, such as the ratio of number of DDD to number of treatment days [5], daily doses per admission (DDDs per admission) [12, 31], and number of days of therapy per 1000 patient days (DOTs per 1000 patient days) [32]. While undoubtedly useful, all of these indicators place emphasis on the difference between administered dosage (prescribed daily doses, PDD) and DDD and ignore the bias of length of stay of different inpatients.

The indicators UR and UD could be suitable for representing antibacterial utilization at the macroscopic level. UR reflects the administration proportion while UD reflects the administration density in a population. When UR remains unchanged, the utilization condition in a population is codetermined by the individual utilization condition of every inpatient. By deriving formulas and extending the meanings of some indicators, such as DUI, the indicator UD/UR was innovatively presented in this article based on the ATC/DDD system. UD/UR synchronously considers the dose and duration of treatment, the two main factors affecting the appropriateness of clinical application of antibiotics, and can sensitively reflect the drug utilization of inpatients receiving antibiotics. Combined with the indicators UR and UD, the proposed indicator could be more suitable for evaluating the clinical appropriateness of antibiotics application than total UD.

$\mathrm{UD} / \mathrm{UR}$ can be applied to the real-time monitoring, comparison, and evaluation of the appropriateness level of antibiotics utilization in hospitals, departments, treatment groups, and individual patients at the macroscopic and microscopic levels. For example, the control goals of antibiotics can be established in different departments at the department level, utilization levels of antibiotics in different treatment groups with the same profession can be compared at the treatment-group level, and abnormal utilization condition can be detected to provide an early warning at the individual-patient level. Various indicators, including UD, UR, and UD/UR, should be considered synthetically when performing the appropriateness evaluation of clinical application of antibiotics to address the limitations of each indicator and produce comprehensive, authentic, precise, and fair evaluation results.

Individualized setting and continuous improvement of the recommended values of UD/UR in clinical practice should be enabled based on the different professional characteristics of various departments or hospitals, such as distribution of disease entities, medication regimens, and mean length of stay. For example, if only one dose of preventative antibiotics (assuming that the frequency of administration is tid) can be used during the period of some operations with type I (clean) incisions, the rational DUI can be assigned as $(1 / 3) /(1 / 3)=1$ and $\mathrm{C} / \mathrm{S}$ as $[(1 / 3) /($ mean length of stay of inpatients receiving antibiotics)]. If no preventative antibiotics can be used during the period of some operations with type I (clean) incisions in principle, the rational DUI and C/S can both be assigned as 0 . The implications of the indicator UD/ UR make it extremely suitable for managing of clinical pathways and charge based on disease entities (diagnosis-related groups, DRGs). It is also useful for the specification and stepwise implementation of the responsibility system of clinical application management of antibiotics.

\section{Conclusions}

UD/UR can sensitively reflect the drug utilization of inpatients receiving antibiotics and be applied at the macroscopic and microscopic levels. This indicator has great practical value and can serve as a reference for evaluating the appropriateness of clinical application of antibiotics.

\section{Abbreviations \\ C/S: ratio of antimicrobial course to length of stay; DDD: Defined daily dose; DUI: Drug utilization index; EF: Elongation factor of length of stay; PDD: Prescribed daily dose; UD: Use density; UD/UR: Ratio of use density to use rate; UR: Use rate}

\section{Funding}

This work was supported by the Fujian Provincial Health and Family Planning Commission (No. 2014-ZQN-JC-15 (design of the study, collection, analysis), and No. 2015-CX-R4 (design of the study, collection, analysis)) and the Science and Technology Department of Fujian Province (No. 2018 R0041 (interpretation of data, writing the manuscript)) of the People's Republic of China.

\section{Availability of data and materials}

The data that support the findings of this study are available from the Hospital Information System of Fujian Medical University Union Hospital (Fuzhou, China) but restrictions apply to the availability of these data, which were used under license for the current study, and so are not publicly available. Data are however available from the authors upon reasonable request and with permission of the hospital. 


\section{Authors' contributions}

BZ: Concept and design, data collection,data interpretation,writing of manuscript. NL: Data interpretation,writing of manuscript. ZJH: Data collection. MBL: Concept and design,data interpretation. All authors read and approved the final manuscript.

\section{Ethics approval and consent to participate}

All study protocols were performed in accordance with and approved by the Committee of Research Ethics of Fujian Medical University Union Hospital (2018KY013, Fuzhou, China). The permission was granted by the participating hospital to access patient information in the Hospital Information System. The consent was not required. Because the IRB thought the primary aim in this article was to verify a new indicator for analyzing the utilization data of antibiotics, the data of individual patients were not directly presented within the manuscript in view of privacy protection and the record numbers of individual patients had been appropriately hidden.

\section{Consent for publication}

Not applicable.

\section{Competing interests}

No conflict of interest exists. This work was supported by the government of Fujian Province of the People's Republic of China. The authors declare that they have no competing interests.

\section{Publisher's Note}

Springer Nature remains neutral with regard to jurisdictional claims in published maps and institutional affiliations.

\section{Author details}

'Department of Pharmacy, Fujian Medical University Union Hospital, 29 Xinquan Road, Gulou District, Fuzhou City 350001, Fujian Province, China. ${ }^{2}$ Department of Information, Fujian Medical University Union Hospital, 29 Xinquan Road, Gulou District, Fuzhou City 350001, Fujian Province, China.

\section{Received: 9 February 2018 Accepted: 28 August 2018}

\section{Published online: 05 September 2018}

\section{References}

1. WHO Collaborating Centre for Drug Statistics Methodology. Guidelines for ATC classification and DDD assignment. Oslo, Norway: Norwegian Inst Publ Hlth; 2009.

2. Raveh $D$, Levy $Y$, Schlesinger $Y$, Greenberg A, Rudensky B, Yinnon AM. Longitudinal surveillance of antibiotic use in the hospital. QJM. 2001;(3):141-52.

3. Beovic B, Kreft S, Seme K, Cizman M. The impact of total control of antibiotic prescribing by infectious disease specialist on antibiotic consumption and cost. J Chemother. 2009;(1):46-51.

4. Porta A, Hsia Y, Doerholt $K$, et al. Comparing neonatal and paediatric antibiotic prescribing between hospitals: a new algorithm to help international benchmarking. J Antimicrob Chemother. 2012;(5):1278-86.

5. Haug JB, Reikvam A. WHO defined daily doses versus hospital-adjusted defined daily doses: impact on results of antibiotic use surveillance. J Antimicrob Chemother. 2013;(12):2940-7.

6. Williams A, Mathai AS, Phillips AS. Antibiotic prescription patterns at admission into a tertiary level intensive care unit in northern India. J Pharm Bioallied Sci 2011; 4: 531-536.

7. Muller A, Monnet DL, Talon D, Hénon T, Bertrand X. Discrepancies between prescribed daily doses and $\mathrm{WHO}$ defined daily doses of antibacterials at a university hospital. Br J Clin Pharmacol. 2006;(5):585-91.

8. Bevilacqua $S$, Demoré B, Boschetti $\mathrm{E}$, et al. Fifteen years of antibiotic stewardship policy in the Nancy teaching hospital. Med Mal Infect. 2011:(10):532-9.

9. Wirtz VJ, Mol PG, Verdijk J, Vander Stichele RH, Taxis K. Use of antibacterial fixed-dose combinations in the private sector in eight Latin American countries between 1999 and 2009. Tropical Med Int Health. 2013;(4):416-25.

10. Mondain V, Lieutier F, Dumas $S$, et al. An antibiotic stewardship program in a French teaching hospital. Med Mal Infect. 2013;(1):17-21.

11. Valcourt K, Norozian F, Lee H, Raszynski A, Torbati D, Totapally BR. Drug use density in critically ill children and newborns: analysis of various methodologies. Pediatr Crit Care Med. 2009;(4):495-9.
12. Kuster $P$, Ruef $C$, Ledergerber $B$, et al. Quantitative antibiotic use in hospitals: comparison of measurements, literature review, and recommendations for a standard of reporting. Infection. 2008;(6):549-59.

13. Kuster SP, Ruef C, Bollinger AK, et al. Correlation between case mix index and antibiotic use in hospitals. J Antimicrob Chemother. 2008;(4):837-42.

14. Akalin S, Kutlu SS, Cirak B, Eskiçorapcı SY, Bagdatli D, Akkaya S. Application of ATC/DDD methodology to evaluate perioperative antimicrobial prophylaxis. Int J Clin Pharm. 2012;(1):120-6.

15. Kritsotakis El, Gikas A. Surveillance of antibiotic use in hospitals: methods, trends and targets. Clin Microbiol Infect. 2006;(8):701-4.

16. The National Health and Family Planning Commission (NHFPC) of the People's Republic of China. Management of Medical Quality and Control Indicators of Tertiary General Hospitals (version 2011). Available at: http:// www.nhfpc.gov.cn/zwgk/wtwj/201304/ 218ca4b1086949ae9261e487e572b299.shtml. Accessed 26 Jan 2011.

17. The National Health and Family Planning Commission (NHFPC) of the People's Republic of China. National Program of Special Renovation Activity on the Clinical Application of Antibiotics in 2012. Available at: http://www. nhfpc.gov.cn/zwgkzt/wsbysj/201203/54251.shtml. Accessed 6 Mar 2012.

18. Ghodse AH, Baigent B, Evans TC, Anderson S. The use of a drug utilisation indicator to monitor psychotropic drug use in hospitals. J Soc Psych. 1987;(1):21-9.

19. Teng L, Xin HW, Blix HS, Tsutani K. Review of the use of defined daily dose concept in drug utilization research in China. Pharmacoepidemiol Drug Saf. 2012:(10):1118-24

20. Zhang L, Li Y, Zeng L, Wang Z. Applying "children defined daily dose" to assess appropriate dose in pediatrics. J Evid Based Med. 2012;(1):2-5.

21. U.S. Food and Drug Administration. ROCEPHIN (ceftriaxone sodium) FOR INJECTION. Available at: https://www.accessdata.fda.gov/drugsatfda_docs/ label/2015/050585s066lbl.pdf Accessed 23 Mar 2015.

22. Andreasen JO, Jensen SS, Schwartz O, Hillerup Y. A systematic review of prophylactic antibiotics in the surgical treatment of maxillofacial fractures. J Oral Maxillofacial Surg. 2006;(11):1664-8.

23. Aufenacker TJ, Koelemay MJ, Gouma DJ, Simons MP. Systematic review and meta-analysis of the effectiveness of antibiotic prophylaxis in prevention of wound infection after mesh repair of abdominal wall hernia. Br J Surg. 2006:(1):5-10.

24. Sanchez-Manuel FJ, Seco-Gil JL. Antibiotic prophylaxis for hernia repair Cochrane Database Syst Rev. 2004, (4): CD003769; PMID: 15495064]. Cochrane Database Syst Rev 2003, 2.

25. Guillot J, Lebel D, Roy H, Ovetchkine P, Bussières JF. Usefulness of defined daily dose and days of therapy in pediatrics and obstetrics-gynecology: a comparative analysis of antifungal drugs (2000-2001, 2005-2006, and 2010-2011). J Pediatr Pharmacol Ther 2014; 3: 196-201.

26. Bansal D, Mangla S, Undela $K$, et al. Measurement of adult antimicrobial drug use in tertiary care hospital using defined daily dose and days of therapy. Indian J Pharm Sci. 2014;3:211-7.

27. Pathak A, Mahadik K, Dhaneria SP, Sharma A, Eriksson B, Lundborg CS Surveillance of antibiotic consumption using the "focus of infection" approach in 2 hospitals in Ujjain, India. PLoS One. 2012;(6):e38641.

28. Eryilmaz EN. An approach to 'dynamic--DDD (defined daily dose) monitoring' to reduce adverse clinical outcomes and increase patient safety: information repositories and event triggers in clinical practice. Stud Health Technol Inform. 2011;166:156-65.

29. Kritsotakis El, Assithianakis P, Kanellos P, Tzagarakis N, loannides MC, Gikas A. Surveillance of monthly antimicrobial consumption rates stratified by patient-care area: a tool for triggering and targeting antibiotic policy changes in the hospital. J Chemother. 2006;(4):394-401.

30. Juhasz Z, Benko R, Matuz M, Viola R, Soos G, Hajdu E. Treatment of acute cystitis in Hungary: comparison with national guidelines and with diseasespecific quality indicators. Scand J Infect Dis. 2013;(8):612-5.

31. de With K, Maier L, Steib-Bauert M, Kern P, Kern WV. Trends in antibiotic use at a university hospital: defined or prescribed daily doses? Patient days or admissions as denominator? Infection. 2006:(2):91-4.

32. Polk RE, Fox C, Mahoney A, Letcavage J, MacDougall C. Measurement of adult antibacterial drug use in 130 US hospitals: comparison of defined daily dose and days of therapy. Clin Infect Dis. 2007;(5):664-70. 